\title{
Respiratory Syncytial Virus Hospitalizations among U.S. Preterm Infants Compared with Term Infants Before and After the 2014 American Academy of Pediatrics Guidance on Immunoprophylaxis: 2012-2016
}

\author{
Mitchell Goldstein, MD ${ }^{1}$ Leonard R. Krilov, MD² Jaime Fergie, MD ${ }^{3}$ Kimmie K. McLaurin, MS \\ Sally W. Wade, MPH ${ }^{5}$ David Diakun, BS ${ }^{6}$ Gregory M. Lenhart, MS ${ }^{6}$ Adam Bloomfield, MD ${ }^{4}$ \\ Amanda M. Kong, $\mathrm{MPH}^{6}$
}

${ }^{1}$ Division of Neonatal Medicine, Loma Linda University Children's Hospital, Loma Linda, California

2 Pediatric Infectious Diseases, Children's Medical Center, NYU Winthrop, Mineola, New York

${ }^{3}$ Department of Pediatric Infectious Disease, Driscoll Children's Hospital, Corpus Christi, Texas

${ }^{4}$ AstraZeneca, Gaithersburg, Maryland

${ }^{5}$ Wade Outcomes Research and Consulting, Salt Lake City, Utah

${ }^{6}$ Truven Health Analytics, an IBM Company, Cambridge, Massachusetts

Am J Perinatol 2018;35:1433-1442.

\begin{abstract}
Address for correspondence Mitchell Goldstein, MD, Loma Linda University Children's Hospital, 11175 Campus Street, Loma Linda, CA 92354 (e-mail: MGoldstein@llu.edu).
\end{abstract}

\begin{abstract}
Keywords

- respiratory syncytial virus

- immunoprophylaxis

- hospitalization rates

- palivizumab

Objective The objective of this study was to compare risk for respiratory syncytial virus (RSV) hospitalizations (RSVH) for preterm infants 29 to 34 weeks gestational age (WGA) versus term infants before and after 2014 guidance changes for immunoprophylaxis (IP), using data from the 2012 to 2016 RSV seasons.

Study Design Using commercial and Medicaid claims databases, infants born between July 1, 2011 and June 30, 2016 were categorized as preterm or term. RSVH during the RSV season (November-March) were identified for infants aged $<6$ months and rate ratios (RRs) for hospitalization comparing preterm and term infants were calculated. Difference-indifference models were fit to evaluate the changes in hospitalization risks in preterm versus term infants from 2012 to 2014 seasons to 2014 to 2016 seasons.

Results In all seasons, preterm infants had higher RSVH rates than term infants. Seasonal RRs prior to the guidance change for preterm wGA categories versus term infants ranged from 1.6 to 3.4. After the guidance change, the seasonal RRs ranged from 2.6 to 5.6. In 2014 to 2016, the risk associated with prematurity of 29 to 34 wGA versus term was significantly higher than in 2012 to $2014(P<0.0001$ for commercial and Medicaid samples).

Conclusion In infants aged $<6$ months, the risk for RSVH for infants 29 to 34 wGA compared with term infants increased significantly after the RSV IP recommendations became more restrictive.
\end{abstract}

Every year in the United States, an estimated 132,000 to 172,000 respiratory syncytial virus (RSV)-associated hospitalizations occur in children aged $<5$ years. ${ }^{1}$ Those at greatest risk for severe RSV disease, including bronchiolitis and pneumonia, are preterm infants, infants aged $<6$ months, and infants with other health conditions, such as chronic received

March 7, 2018 accepted after revision April 26, 2018 published online June 19, 2018
DOI https://doi.org/ 10.1055/s-0038-1660466. ISSN 0735-1631.
Copyright $\odot 2018$ by Thieme Medical Publishers, Inc., 333 Seventh Avenue, New York, NY 10001, USA. Tel: +1(212) 584-4662.
License terms

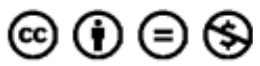


lung disease (CLD) of prematurity (CLDP), congenital heart disease (CHD), and immunodeficiencies. ${ }^{2-7}$ Preterm infants are vulnerable to RSV infection because of small lung volumes and narrow airway diameters as well as an immature immune system when compared with term infants. ${ }^{8-11}$

Although no treatment for severe RSV disease exists, the risk of severe disease can be lowered with palivizumab immunoprophylaxis (IP). ${ }^{12}$ Palivizumab, a monoclonal antibody, is approved for the prevention of severe RSV infection in children with bronchopulmonary dysplasia (BPD)/CLD, children with hemodynamically significant CHD, and children born at $\leq 35$ weeks gestational age (wGA). ${ }^{12}$

In the United States, the American Academy of Pediatrics (AAP) Committee on Infectious Diseases (COID) issues guidance on the use of RSV IP that is updated periodically. In 2009 through 2013, the AAP recommended the use of RSV IP in the following groups based on prematurity: infants born at $\leq 28$ wGA and aged $<12$ months at the start of the RSV season, infants born at 29 to 31 wGA and aged $<6$ months at the start of the RSV season, and infants born at 32 to 34 wGA and aged $<3$ months at the start of the RSV season who attended day care and/or had school-aged siblings. ${ }^{13}$ In 2014 , the guidance was modified to recommend against the use of RSV IP in infants born at 29 to 34 wGA unless the infant had an underlying health condition, including BPD/CLD or CHD. ${ }^{14}$

Several studies have assessed the impact of the guidance change on RSV IP rates or on RSV hospitalization rates. ${ }^{15-20} \mathrm{~A}$ decrease in RSV IP was demonstrated in a large analysis, ${ }^{20}$ and most studies of RSV hospitalizations have found increased RSV morbidity in the season after the guidance change, particularly among infants aged $<3$ months. ${ }^{15-17,20}$ To date, published research on the impact of the AAP guidance change has been focused on the 2014-2015 RSV season. More recent trends, including the 2015-2016 RSV season, have not yet been described in large, geographically broad populations of infants with either commercial or public insurance coverage.

The primary objectives of this study were to compare (1) the proportion of infants receiving RSV IP and (2) RSV hospitalization rates in infants 29 to 34 wGA relative to term infants in the two RSV seasons after the guidance change (2014-2015 and 2015-2016) to the two RSV seasons preceding the guidance change (2012-2013 and 2013-2014) among infants aged $<6$ months. Two seasons were combined to minimize the impact of seasonal variability in RSV circulation and attack rates. A secondary objective was to describe RSV hospitalization costs for preterm and term infants before and after the guidance change.

\section{Methods}

\section{Data Sources}

This analysis was conducted using the Truven Health MarketScan Commercial and Multi-State Medicaid databases. These databases have been used in several previous RSV analyses. $^{20-25}$ Both databases contain enrollment information, inpatient medical, outpatient medical, and outpatient phar- macy claims. The commercial database includes enrollees from self-insured employers and commercial health plans, and the Medicaid database includes enrollees from several geographically diverse states. The data were previously collected and statistically deidentified, and are compliant with the conditions set forth in Sections 164.514(a)-(b)(1)ii of the Health Insurance Portability and Accountability Act of 1996 Privacy Rule; therefore, approval from an Institutional Review Board was not sought.

\section{Patient Selection}

Using International Classification of Diseases, Ninth Revision, Clinical Modification (ICD-9-CM) and International Classification of Diseases, Tenth Revision, Clinical Modification (ICD-10-CM) diagnosis codes and Diagnosis-Related Group (DRG) codes on inpatient claims, we selected infants born from July 1, 2011, through June 30, 2016, in both databases. Infants were required to have been discharged alive from their birth hospitalization. Next, infants were classified into risk cohorts using diagnoses, DRGs, procedures, and medications appearing in their medical and pharmacy claims during the first 90 days of life. To ensure mutually exclusive cohorts, we applied the following hierarchy when categorizing the infants: (1) infants with complex, rare medical conditions (e.g., infants with evidence of cystic fibrosis, immunodeficiency, congenital anomalies of the respiratory system, neuromuscular, immunological or genetic conditions, or organ transplants), (2) infants with CLDP, (3) infants with CHD, (4) infants born at $<29$ wGA, (5) infants born at 29 to $30 \mathrm{wGA},(6)$ infants born at 31 to 32 wGA, (7) infants born at 33 to 34 wGA, (8) infants born at 35 to 36 wGA, (9) infants born at $>36$ wGA (term) with major health problems, (10) infants born at $>36$ wGA without major health problems, (11) other preterm infants with unknown wGA, and (12) other infants with unknown wGA. ${ }^{20}$

In this study, we analyzed preterm infants born at 29 to 34 wGA-the group affected by the 2014 change in RSV IP guidance-and term infants without major health problems, who were not affected by the guidance change. Infants in other cohorts were excluded.

\section{Infant Follow-up}

Infants were followed from birth through the earliest of the following events: end of the first 6 months of life, death, or end of continuous enrollment in the database. Infant persontime was captured overall and by groups based on chronological age (i.e., aged $<3$ and $3-<6$ months) at any point during the RSV season. The total number of days that an infant contributed during the RSV season was divided by 151 days (the number of days during the RSV season) to present follow-up as infant-seasons. As infants were followed for up to 6 months, they may have contributed time to more than one chronological age group for certain outcomes, but could contribute to only one RSV season for the primary outcomes. An infant may have contributed to two time periods for the secondary outcome of RSV hospitalization costs. 


\section{Outcomes}

The primary outcomes were RSV IP utilization and risk of RSV hospitalizations occurring from November to March (the RSV season) in the 2012-2013, 2013-2014, 2014-2015, and 20152016 RSV seasons. For the primary analysis, outcomes were assessed over multiple seasons combined (2012 to 2014 and 2014 to 2016). RSV IP was identified in outpatient medical and pharmacy claims by drug codes (NDC) or administration codes (current procedural terminology or Healthcare Common Procedure Coding System). Inpatient doses could not be captured in these databases. RSV hospitalizations were identified by evaluating inpatient claims for an ICD-9-CM (079.6, 466.11, 480.1) or ICD-10-CM diagnosis (B974, J205, J121, J210) indicating RSV in any position on the claim. Hospitalizations occurring $<2$ days after birth hospitalization discharge were not analyzed to limit misclassification of transfers and readmissions from birth hospitalizations.

All-cause bronchiolitis hospitalizations were also captured during the RSV seasons to assess the broader potential absolute effects of a guidance change and to determine if changes in RSV hospitalization rates may have been due to changes in coding on claims. All-cause bronchiolitis hospitalizations comprised hospitalizations with a diagnosis of RSV bronchiolitis and unspecified bronchiolitis. Unspecified bronchiolitis hospitalizations were identified by diagnosis of ICD-9-CM 466.19 or ICD-10-CM J211, J218, J219 and no diagnoses of influenza, bacterial pneumonia, or other viral pathogens within 3 days of admission. Again, hospitalizations occurring $<2$ days after birth hospitalization discharge were not included in this analysis.

For the secondary objective, RSV hospitalization costs were captured for all RSV hospitalizations from July 1, 2012, through June 30, 2016, to include all RSV hospitalization-related costs. Costs were based on the paid amount on the identified inpatient claims, which included insurer-paid and patient out-of-pocket costs and were adjusted to 2016 U.S. dollars.

\section{Statistical Analyses}

RSV IP utilization was reported as the count and proportion of infants with at least one outpatient claim for RSV IP during follow-up. Both the absolute and the percentage decrease from the 2012 to 2014 RSV seasons to the 2014 to 2016 RSV seasons were calculated. The proportions of infants with RSV IP in the two time periods were compared using chi-squared tests.

RSV hospitalization rates were calculated as the number of RSV hospitalizations per 100 infant-seasons. All-cause bronchiolitis hospitalization rates were calculated in the same manner. To investigate the additional RSV and all-cause bronchiolitis hospitalization risks due to preterm birth after the change in palivizumab recommendations compared with prior years, we first calculated unadjusted rate ratios (RRs) comparing the rate of hospitalizations among preterm infants to that of term infants by season among infants aged $<6$ months. The calculation of the RRs within each season allowed us to account for the seasonal variability in RSV circulation and attack rates that may cause variation in absolute hospitalization rates. RRs for risk of hospitalization were also calculated for the combined 2012 to 2014 and the combined 2014 to 2016 RSV seasons.

Next, difference-in-difference models were fit, controlling for gestational age at birth, chronological age during the RSV season, and sex. As the outcomes modeled (hospitalization events) are counts, we used generalized linear models with Poisson's error distribution, log link, and a log offset term to represent exposure. The difference-in-difference effect estimate was calculated as:

Rate for preterm infants in 2014 to 2016/rate for preterm infants in 2012 to 2014

Rate for term infants in 2014 to 2016 /rate for term infants in 2012 to 2014

$P$-values of $<0.05$ were considered statistically significant.

For RSV hospitalization costs, mean and median costs of RSV hospitalizations are presented stratified by gestational age (29-34 wGA vs. term) and by chronologic age at the time of hospital admission. Costs for RSV hospitalizations occurring between July 2012 and June 2014 and costs for RSV hospitalizations occurring between July 2014 and June 2016 are reported separately.

\section{Results}

\section{Characteristics of Infants}

There were 1,134,164 commercially insured infants and 1,448,990 Medicaid-insured infants born and discharged alive in the two databases from July 1, 2011, through June 30, 2016 (-Supplementary Table 1, available in the online version). After creating cohorts for infants with rare, complex medical conditions, CLDP, and CHD, in the commercial sample, 3.0\% ( $n=33,667)$ were 29 to 34 wGA and $59.0 \%$ ( $n=668,619)$ were term without major health problems; in the Medicaid sample, $3.5 \%(n=51,439)$ were 29 to 34 wGA and $62.7 \%(n=908,594)$ were term without major health problems. These infants were included in the analysis. In the 2012 to 2014 RSV seasons, the preterm infants 29 to 34 wGA aged $<6$ months contributed 5,803 and 9,582 infant-seasons in the commercial and Medicaid analyses, respectively, whereas term infants without major health problems aged $<6$ months contributed 134,655 and 194,682 infant-seasons, respectively (-Supplementary Table 2, available in the online version). In the 2014 to 2016 RSV seasons, preterm infants aged $<6$ months contributed 4,440 and 9,317 infant-seasons in the commercial and Medicaid analyses, respectively, whereas term infants without major health problems aged $<6$ months contributed 97,546 and 178,214 infant-seasons, respectively (-Supplementary Table 2, available in the online version). Demographic characteristics for infants who contributed person-time when aged $<6$ months during the 2012 to 2016 RSV seasons can be found in -Supplementary Table 3, available in the online version.

\section{RSV Immunoprophylaxis}

Prior to the 2014 guidance change, RSV IP rates within each wGA category ranged from 25.5 to $49.8 \%$ among infants $\leq 32$ wGA who were aged $<6$ months during the RSV season. 


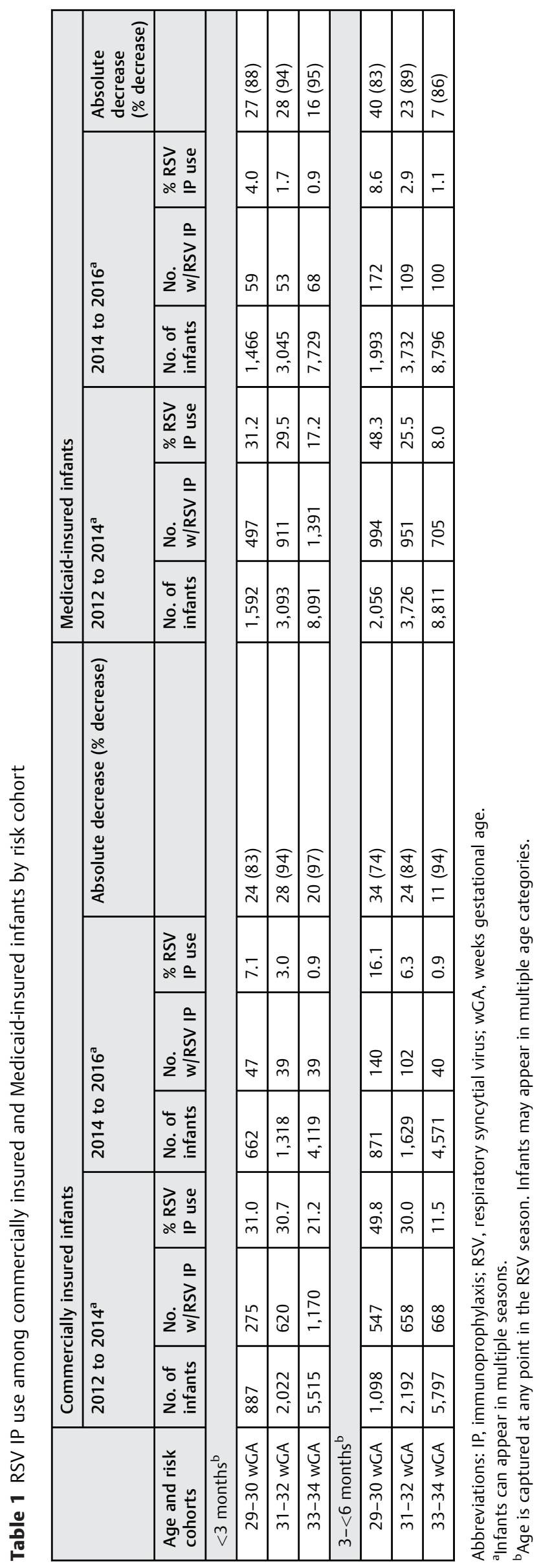

Rates were lower among infants born at 33 to 34 wGA. Following the 2014 guidance change, the proportion of infants receiving RSV IP decreased significantly for all preterm age groups in both databases (all $P<0.0001$ within the commercial and Medicaid populations). In the 2014 to 2016 seasons, rates within each wGA category ranged from 1.7 to $16.1 \%$ among infants $\leq 32 \mathrm{wGA}$ who were aged $<6$ months during the RSV season. The percentage decrease in the proportion of infants with IP was $74 \%$ or more for all groups analyzed (-Table $\mathbf{1}$ ). Before and after the guidance change, the proportion of term infants with RSV IP was less than $0.02 \%$.

\section{RSV Hospitalizations Rates}

Among commercially insured preterm infants 29 to 34 wGA and term infants aged $<6$ months, we identified $1,586 \mathrm{RSV}$ hospitalizations during the 140,459 infant-seasons contributed during the 2012 to 2014 RSV seasons and 1,028 RSV hospitalizations during the 101,986 infant-seasons contributed during the 2014 to 2016 RSV seasons. Among Medicaidinsured preterm infants 29 to $34 \mathrm{wGA}$ and term infants aged $<6$ months, there were 4,122 RSV hospitalizations in the 204,264 infant-seasons contributed during 2012 to 2014 RSV seasons and 2,799 RSV hospitalizations in the 187,530 infant-seasons during the 2014 to 2016 RSV seasons.

In all seasons evaluated, preterm infants aged $<6$ months had higher rates of RSV hospitalization than term infants aged $<6$ months, and therefore, all unadjusted RRs comparing preterm to term infants are $>1$. There was an increase in the RRs across time, specifically in the seasons after the guidance change (-Fig. 1A). Seasonal RRs prior to the guidance change for the wGA categories versus term infants ranged from 1.6 to 3.4 ; however, after the guidance change, the seasonal RRs ranged from 2.6 to 5.6. For commercially insured infants, the RRs of RSV hospitalization for preterm infants versus term infants increased from the combined 2012 to 2014 seasons to the combined 2014 to 2016 seasons, with the largest increase found in infants 29 to 30 wGA and aged $<3$ months (RR vs. term $=1.0$ in 2012 to 2014 vs. RR vs. term $=4.3$ in 2014 to 2016 ; - Fig. 2A). The differences in RRs between the two time periods were generally smaller among Medicaid-insured infants (- Fig. 2B).

In difference-in-difference models, the increase in risk of RSV hospitalization associated with prematurity of 29 to 34 wGA compared with term in the two seasons after the guidance change was double $(2.00, P<0.0001)$ the increase in risk associated with prematurity in the two seasons prior to the recommendation change for commercially insured infants and $46 \%$ higher $(R R=1.46, P<0.0001)$ for Medicaid-insured infants in the postguidance change period compared with the preguidance change period (-Table 2). The amount of increase in risk associated with the guidance change is positively correlated with increasing degree of prematurity, with RRs being highest among infants born at 29 to 30 wGA.

Absolute hospitalization rates in the combined 2012 to 2014 seasons and combined 2014 to 2016 seasons provide insight into the absolute rates of disease by infant subgroup before and after the 2014 guidance change (- Supplementary 

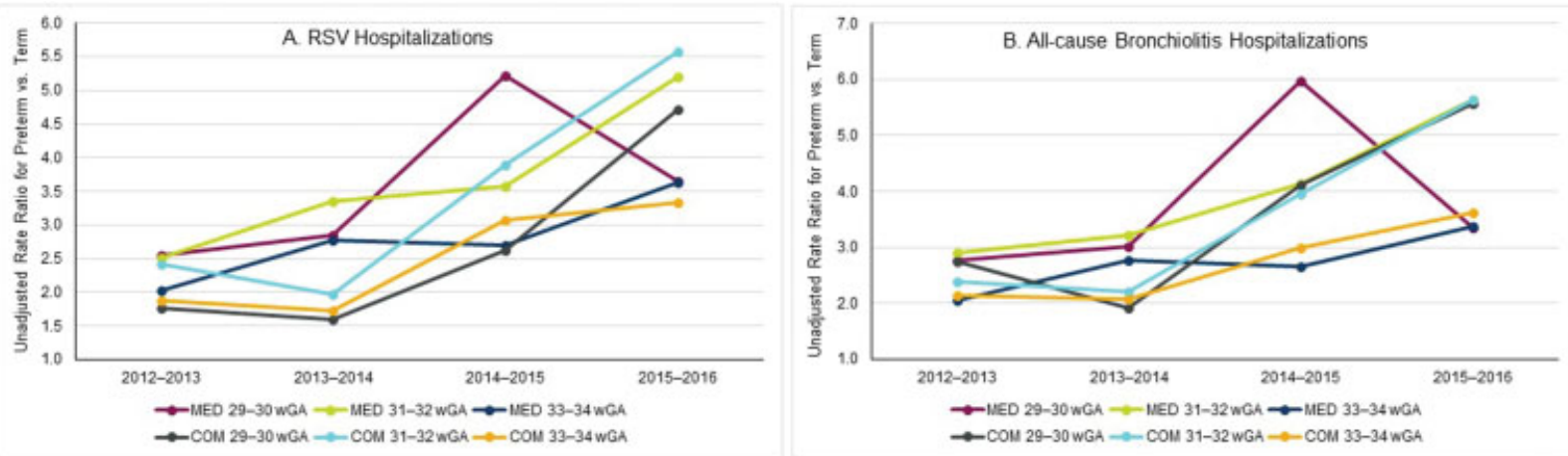

Fig. 1 (A, B) Unadjusted rate ratios for RSV hospitalization and all-cause bronchiolitis hospitalization comparing preterm to term infants by season, among infants aged $<6$ months. COM, commercial; MED, Medicaid; RSV, respiratory syncytial virus; wGA, weeks gestational age.
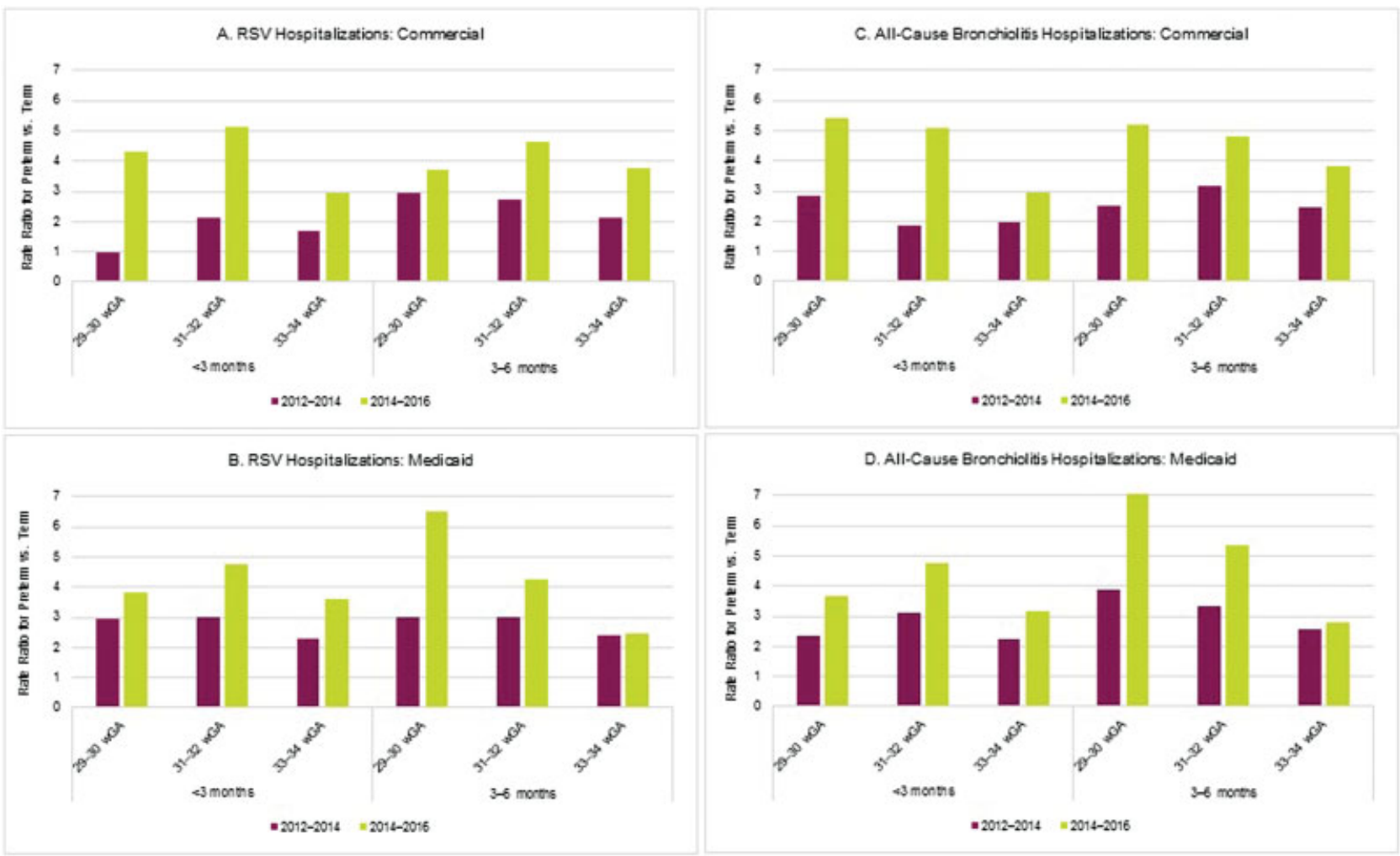

Fig. 2 (A-D) Unadjusted rate ratios for RSV hospitalization and all-cause bronchiolitis hospitalization comparing preterm infants to term infants by season. RSV, respiratory syncytial virus; wGA, weeks gestational age.

Table 4, available in the online version). There was an increase in absolute rates among all preterm infant groups in the commercially insured sample, with a 10 to $13 \%$ decrease among term infants. Among Medicaid-insured infants, there were smaller increases among most preterm infant groups with no increase in some groups, but with a 27 to $28 \%$ decrease in rates among term infants. Absolute rates were higher among Medicaid-insured infants than commercially insured infants.

\section{All-Cause Bronchiolitis Hospitalizations}

Among commercially insured preterm infants 29 to 34 wGA and term infants aged $<6$ months, we identified 2,347 all- cause bronchiolitis hospitalizations during the 140,459 infantseasons contributed during the 2012 to 2014 RSV seasons and 1,503 all-cause bronchiolitis hospitalizations during the 101,986 infant-seasons contributed during the 2014 to 2016 RSV seasons. Among Medicaid-insured preterm infants 29 to 34 wGA and term infants aged $<6$ months, there were 5,960 all-cause bronchiolitis hospitalizations in the 204,264 infantseasons contributed during 2012 to 2014 RSV seasons and 3,996 all-cause bronchiolitis hospitalizations in the 187,530 infant-seasons during the 2014 to 2016 RSV seasons.

Results for all-cause bronchiolitis hospitalizations were similar to RSV hospitalizations. Preterm infants aged $<6$ months had higher rates of all-cause bronchiolitis 
hospitalization than term infants aged $<6$ months in all four seasons evaluated. As noted with RSV hospitalizations, there was a general increase in the RRs for all-cause bronchiolitis hospitalizations across time, specifically in the seasons after the guidance change ( $\mathbf{- F i g}$. 1B). Seasonal RRs prior to the guidance change for the wGA categories versus term infants ranged from 1.9 to 3.2; however, after the guidance change, the seasonal RRs for all-cause bronchiolitis hospitalizations ranged from 2.7 to 6.0. When combining the 2012 to 2014 and 2014 to 2016 RSV seasons, RRs for all-cause bronchiolitis hospitalizations were higher after the guidance change in both samples (-Fig. 2C, D).

In difference-in-difference models, the increase in risk of all-cause bronchiolitis hospitalization associated with prematurity of 29 to 34 wGA compared with term in the two seasons after the guidance change was $89 \%$ higher $(\mathrm{RR}=1.89, P<0.0001)$ than the increase in risk associated with prematurity in the two seasons prior to the guidance change for commercially insured infants and $45 \%$ higher (RR $=1.45, P<0.0001)$ for Medicaid-insured infants postguidance change compared with preguidance (-Table $\mathbf{2}$ ).

Absolute rates of all-cause bronchiolitis hospitalizations increased after the guidance change for preterm infants, whereas rates for term infants decreased (-Supplementary Table 4, available in the online version).

\section{RSV Hospitalization Costs}

Among infants with an RSV hospitalization, average costs for preterm infants were higher than average costs for term infants both before and after the guidance change (-Table 3 ). In the time period after the guidance change, the mean costs for RSV hospitalizations for infants hospitalized when aged $<3$ months were more than twice as high for preterm infants than for term infants (Commercial: $\$ 41,104$ [median $\$ 16,792$ ] vs. $\$ 17,597$ [\$10,494]; Medicaid: $\$ 24,049$ [median $\$ 8,521$ ] vs. $\$ 10,987$ [median \$4,257]). Costs were also higher for infants hospitalized when aged $<3$ months than those hospitalized when aged 3 to $<6$ months. RSV hospitalization costs tended to increase from 2012 to 2014 to 2014 to 2016, with the largest increases noted for preterm infants 29 to 34 wGA.

\section{Discussion}

This analysis of commercially insured and Medicaid-insured infants aged $<6$ months found that the proportion of preterm infants 29 to 34 wGA who received RSV IP decreased significantly in the two seasons after the AAP guidance change. Concurrently, the already elevated risk for RSV hospitalization for preterm infants compared with term infants increased significantly in the seasons after the guidance change. For those infants who are hospitalized with RSV, the costs of RSV hospitalizations are also higher for preterm infants compared with term infants.

This study confirms that the decrease in RSV IP in the United States among otherwise healthy preterm infants reported by Kong et al in the first season after the guidance change continued in the subsequent season. ${ }^{20}$ In that analysis, RSV IP rates also decreased among preterm infants between 6 and 12 months old, although palivizumab utilization was less common among infants in that age group. ${ }^{20}$ Palivizumab IP has been shown to significantly decrease the risk for RSV hospitalization in three clinical trials. ${ }^{26-28}$ Recently, Anderson et al conducted an effectiveness study using a multicenter, testnegative, inverse propensity score weighted design to assess the real-world effectiveness of palivizumab. ${ }^{29}$ They found that palivizumab was $74 \%$ effective (95\% confidence interval [CI]: 56-85\%) in preventing RSV-related hospitalizations among infants born at 29 to $35 \mathrm{wGA}$ and with chronologic age $<6$ months without CLDP or CHD, after controlling for differences between infants who did and did not receive palivizumab. ${ }^{29}$

Table 2 Change in RSV hospitalization risk and all-cause bronchiolitis risk from 2012 to 2014 to 2014 to 2016 among preterm infants aged $<6$ months compared with change in risk among term infants aged $<6$ months

\begin{tabular}{|c|c|c|c|c|c|c|c|c|}
\hline \multirow[t]{2}{*}{ wGA } & \multicolumn{4}{|c|}{ Commercially insured infants } & \multicolumn{4}{|c|}{ Medicaid-insured infants } \\
\hline & $\begin{array}{l}\text { Difference- } \\
\text { in-difference } \\
\text { for RSV } \\
\text { hospitalization } \\
(95 \% \mathrm{Cl})\end{array}$ & $P$-value & $\begin{array}{l}\text { Difference- } \\
\text { in-difference } \\
\text { for all-cause } \\
\text { bronchiolitis } \\
\text { hospitalization } \\
(95 \% \mathrm{Cl})\end{array}$ & $P$-value & $\begin{array}{l}\text { Difference- } \\
\text { in-difference } \\
\text { for RSV } \\
\text { hospitalization } \\
(95 \% \mathrm{Cl})\end{array}$ & $P$-value & $\begin{array}{l}\text { Difference- } \\
\text { in-difference } \\
\text { for all-cause } \\
\text { bronchiolitis } \\
\text { hospitalization } \\
(95 \% \mathrm{Cl})\end{array}$ & $P$-value \\
\hline $29-34$ & $\begin{array}{l}2.00 \\
(1.42-2.81)\end{array}$ & $<0.0001$ & $\begin{array}{l}1.89 \\
(1.46-2.43)\end{array}$ & $<0.0001$ & $\begin{array}{l}1.46 \\
(1.24-1.73)\end{array}$ & $<0.0001$ & $\begin{array}{l}1.45 \\
(1.26-1.66)\end{array}$ & $<0.0001$ \\
\hline $29-30$ & $\begin{array}{l}2.11 \\
(0.94-4.72)\end{array}$ & 0.0700 & $\begin{array}{l}2.10 \\
(1.18-3.75)\end{array}$ & 0.0119 & $\begin{array}{l}1.61 \\
(1.11-2.32)\end{array}$ & 0.0120 & $\begin{array}{l}1.54 \\
(1.13-2.09)\end{array}$ & 0.0056 \\
\hline $31-32$ & $\begin{array}{l}2.15 \\
(1.33-3.48)\end{array}$ & 0.0018 & $\begin{array}{l}2.06 \\
(1.39-3.04)\end{array}$ & 0.0003 & $\begin{array}{l}1.48 \\
(1.15-1.91)\end{array}$ & 0.0024 & $\begin{array}{l}1.58 \\
(1.29-1.93)\end{array}$ & $<0.0001$ \\
\hline $33-34$ & $\begin{array}{l}1.76 \\
(1.27-2.44)\end{array}$ & 0.0006 & $\begin{array}{l}1.55 \\
(1.20-2.00)\end{array}$ & 0.0009 & $\begin{array}{l}1.32 \\
(1.10-1.58)\end{array}$ & 0.0033 & $\begin{array}{l}1.25 \\
(1.07-1.46)\end{array}$ & 0.0044 \\
\hline
\end{tabular}

Abbreviations: $\mathrm{Cl}$, confidence interval; RSV, respiratory syncytial virus; wGA, weeks gestational age.

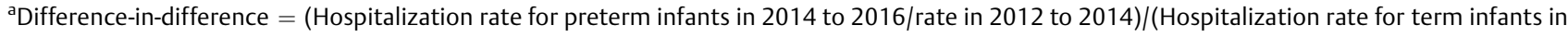
2014 to 2016/rate in 2012 to 2014). 
Table 3 Costs of RSV hospitalizations among preterm and term infants aged $<6$ months

\begin{tabular}{|c|c|c|c|c|c|c|c|c|}
\hline & \multicolumn{4}{|c|}{ Commercially insured infants } & \multicolumn{4}{|c|}{ Medicaid-insured infants } \\
\hline & \multicolumn{2}{|c|}{$\begin{array}{l}\text { July } 2012 \text { to June } \\
2014\end{array}$} & \multicolumn{2}{|c|}{$\begin{array}{l}\text { July } 2014 \text { to June } \\
2016\end{array}$} & \multicolumn{2}{|c|}{$\begin{array}{l}\text { July } 2012 \text { to June } \\
2014\end{array}$} & \multicolumn{2}{|c|}{$\begin{array}{l}\text { July } 2014 \text { to June } \\
2016\end{array}$} \\
\hline & $\begin{array}{l}N / \text { Mean/ } \\
\text { median }\end{array}$ & SD & $\begin{array}{l}\text { N/Mean/ } \\
\text { median }\end{array}$ & SD & $\begin{array}{l}\text { N/Mean/ } \\
\text { median }\end{array}$ & SD & $\begin{array}{l}\text { N/Mean/ } \\
\text { median }\end{array}$ & SD \\
\hline \multicolumn{9}{|l|}{ 29-34 wGA, $<3$ months } \\
\hline $\begin{array}{l}\text { No. of infants hospitalized } \\
\text { with RSV }\end{array}$ & 73 & & 90 & & 286 & & 289 & \\
\hline No. of RSV hospitalizations & 82 & & 92 & & 304 & & 301 & \\
\hline $\begin{array}{l}\text { RSV hospitalization costs }{ }^{\mathrm{a}} \\
\text { (mean, SD) }\end{array}$ & $\$ 36,347$ & $\$ 49,124$ & $\$ 41,104$ & $\$ 65,956$ & $\$ 16,598$ & $\$ 29,339$ & $\$ 24,049$ & $\$ 45,949$ \\
\hline Median & $\$ 14,590$ & & $\$ 16,792$ & & $\$ 6,746$ & & $\$ 8,521$ & \\
\hline \multicolumn{9}{|l|}{ Term, $<3$ months } \\
\hline $\begin{array}{l}\text { No. of infants hospitalized } \\
\text { with RSV }\end{array}$ & 1,089 & & 609 & & 2,540 & & 1,689 & \\
\hline No. of RSV hospitalizations & 1,117 & & 631 & & 2,653 & & 1,747 & \\
\hline $\begin{array}{l}\text { RSV hospitalization costs } \\
\text { (mean, SD) }\end{array}$ & $\$ 14,550$ & $\$ 26,476$ & $\$ 17,597$ & $\$ 27,220$ & $\$ 9,226$ & $\$ 30,519$ & $\$ 10,897$ & $\$ 34,463$ \\
\hline Median & $\$ 8,947$ & & $\$ 10,494$ & & $\$ 3,797$ & & $\$ 4,257$ & \\
\hline \multicolumn{9}{|l|}{ 29-34 wGA, 3-<6 months } \\
\hline $\begin{array}{l}\text { No. of infants hospitalized } \\
\text { with RSV }\end{array}$ & 63 & & 67 & & 200 & & 182 & \\
\hline No. of RSV hospitalizations & 65 & & 71 & & 209 & & 190 & \\
\hline $\begin{array}{l}\text { RSV hospitalization costs } \\
\text { (mean, SD) }\end{array}$ & $\$ 13,782$ & $\$ 16,861$ & $\$ 21,549$ & $\$ 28,278$ & $\$ 8,256$ & $\$ 19,360$ & $\$ 15,755$ & $\$ 93,027$ \\
\hline Median & $\$ 8,679$ & & $\$ 11,440$ & & $\$ 4,365$ & & $\$ 3,749$ & \\
\hline \multicolumn{9}{|l|}{ Term, 3-<6 months } \\
\hline $\begin{array}{l}\text { No. of infants hospitalized } \\
\text { with RSV }\end{array}$ & 525 & & 344 & & 1,375 & & 902 & \\
\hline No. of RSV hospitalizations & 538 & & 350 & & 1,412 & & 929 & \\
\hline $\begin{array}{l}\text { RSV hospitalization costs } \\
\text { (mean, SD) }\end{array}$ & $\$ 11,951$ & $\$ 16,263$ & $\$ 14,293$ & $\$ 20,953$ & $\$ 5,500$ & $\$ 11,379$ & $\$ 6,554$ & $\$ 10,346$ \\
\hline Median & $\$ 7,901$ & & $\$ 8,888$ & & $\$ 3,394$ & & $\$ 3,703$ & \\
\hline
\end{tabular}

Abbreviations: RSV, respiratory syncytial virus; SD, standard deviation; wGA, weeks gestational age.

${ }^{a}$ Hospitalizations with $\$ 0$ cost were excluded.

Preterm infants and young infants were at greater risk for RSV hospitalization than term infants, which is consistent with other analyses. ${ }^{3,6,7,30,31}$ Preterm infants who do not receive RSV IP, especially those at young chronologic ages, are particularly vulnerable to severe RSV infection. Preterm infants have small lung volumes, reduced lung surface area, narrower airways, increased air space wall thickness, and an immature immune system. ${ }^{8-11}$ Infants of younger chronologic age may be at higher risk for similar reasons, that is, their narrower airways and lack of a proficient immune system, as well as contact with other young children. ${ }^{32}$ In the SENTINEL1 study, earlier gestational age and younger chronologic age were associated with increased rates of intensive care unit admission and invasive mechanical ventilation among preterm infants 29 to $35 \mathrm{wGA}$ and aged $<12$ months who were hospitalized with RSV and did not receive RSV IP. ${ }^{15}$ These interventions are associated with greater health care costs. ${ }^{15}$ As demonstrated in our analysis and by an earlier study using data prior to the guidance change, ${ }^{24}$ when limiting to the subset of infants with an RSV infection, health care costs for RSV hospitalizations among preterm infants and those aged $<3$ months were greater than costs among term and infants aged $\geq 3$ months.

When comparing the risk of RSV hospitalization for infants 29 to 34 wGA relative to term infants in the two seasons after the guidance change to the two seasons prior to the guidance change, the risk for RSV hospitalization associated with prematurity increased. This means that the relative difference in risk between preterm and term infants has increased following the 2014 AAP recommendations to a level consistent with the established effectiveness of palivizumab. To our knowledge, this is the first study to analyze the impact of the guidance by evaluating the relative risk of preterm versus term infants, an 
approach that considers and adjusts for overall season severity and secular trends in bronchiolitis admission practices. This comparison also addresses, at least in part, the reason for the guidance change. According to the COID in 2014, preterm infants did not have an increased risk of RSV hospitalization compared with term infants. ${ }^{33,34}$ This study demonstrates that preterm infants do have a higher risk compared with term infants and that risk has increased since the guidance change. As decreased emphasis is given to providing the indicated prophylaxis, up to $75 \%$ of the at-risk population will not be covered. Although the AAP 2014 COID policy attempts to indemnify this practice, it is increasingly clear that patients, hospitals, and insurers will ultimately pay the price for inadequate prophylaxis for the most at-risk populations. Despite the fact that RSV affects infants born at term, it is important to identify this pattern of increased admission secondary to deficient policy as opposed to a change in seasonality or virulence of RSV across all gestations.

Earlier published analyses have focused on RSV hospitalization counts by age group, proportion of infants hospitalized, and changes in the intensity of care required for infants hospitalized with RSV. Most found an increase in RSV hospitalizations and morbidity after the 2014 guidance change. Both Rajah et al and Blake et al described increases in RSV-related morbidity among preterm infants at single institutions. ${ }^{16,17}$ Using the same MarketScan Commercial and Medicaid databases and the patient selection methods described in this article, Kong et al reported increases in rates of RSV hospitalization in the 2014-2015 RSV season among infants 29 to 34 wGA. $^{20}$ Two analyses, one by Grindeland et al and one by Farber, did not report increases in RSV hospitalization rates or severity; however, the studies did not specifically examine preterm infants, ${ }^{18}$ lacked sufficient sample sizes, ${ }^{18,35}$ or examined populations in which RSV IP was known to have continued after the guidance change. ${ }^{19,36}$

This analysis has limitations. First, the claims databases did not contain laboratory results, and therefore, we relied on diagnoses on inpatient medical claims to identify RSV hospitalizations. RSV-coded hospitalizations appear to be specific for true RSV disease, as a previous analysis reported high concordance between RSV diagnosis codes in the emergency room setting and positive RSV tests. ${ }^{37}$ Despite their specificity, RSV-coded hospitalization rates are known to underestimate the true rate of RSV hospitalizations because routine virologic testing is not recommended by the $\mathrm{AAP}^{38}$ and without testing, RSV diagnosis codes may not be used on inpatient claims. Our analysis of all-cause bronchiolitis showed that the risk of all-cause bronchiolitis hospitalizations for premature infants relative to term infants increased after the guidance change regardless of the RSV diagnosis. Second, inpatient medication use is not included in claims, so inpatient utilization of palivizumab was not captured. Because of this, the proportion of infants with RSV IP reported in this study may be underestimated because preterm infants may have received palivizumab in the hospital. An increase in predischarge inpatient dosing of palivizumab in 2015-2016 could explain the decline in RSV hospitalizations among 29 to 30
wGA infants relative to 2014-2015. Third, there were a small number of infants in some gestational age groups. Therefore, the results combining infants born at 29 to 34 wGA are more robust than the results for smaller gestational age groupings (which did not reach statistical significance in some cases). Finally, it is possible that outlier seasons for respiratory diseases could have biased our results. However, we combined two seasons prior to the guidance change and two seasons after the guidance change to minimize any bias due to seasonal variation.

In conclusion, we found that the proportion of infants with outpatient use of RSV IP decreased significantly in infants 29 to 34 wGA and aged $<6$ months in the 2014 to 2016 RSV seasons compared with the 2012 to 2014 RSV seasons. Concurrently, this study demonstrated an increase in risk of RSV hospitalization for preterm infants born at 29 to 34 wGA and aged $<6$ months relative to term infants aged $<6$ months in the combined 2014 to 2016 RSV seasons compared with the 2012 to 2014 RSV seasons. In addition, costs of RSV hospitalizations were shown to be substantially higher for infants 29 to $34 \mathrm{wGA}$ and aged $<6$ months compared with term infants, with the highest costs among infants 29 to 34 wGA and aged $<3$ months after the guidance change.

\section{Note}

This work was presented as a poster titled "Respiratory syncytial virus hospitalization rates and costs among fullterm and preterm infants before and after implementation of the 2014 American Academy of Pediatrics guidance on immunoprophylaxis" at AMCP Nexus 2017, Dallas, TX, October 16-19, 2017, and was presented as a poster titled "RSV Hospitalizations Before and Two Seasons After the 2014 American Academy of Pediatrics Guidance on RSV Immunoprophylaxis" at the 2018 Pediatric Academic Societies Annual Meeting, Toronto, Canada, May 5-8, 2018.

\section{Funding}

This analysis was funded by AstraZeneca.

\section{Conflict of Interest}

Kimmie K. McLaurin and Adam Bloomfield are employees of AstraZeneca; David Diakun, Gregory M. Lenhart, and Amanda M. Kong are employees of Truven Health Analytics, an IBM Company, and Sally W. Wade is a consultant to Truven Health Analytics, which was contracted by AstraZeneca for data analyses and writing support; Leonard $\mathrm{R}$. Krilov has received grant/research support from AstraZeneca/MedImmune; and Mitchell Goldstein and Jaime Fergie have received grant/research support from AstraZeneca/MedImmune and are members of the AstraZeneca Speakers' Bureau.

\section{Acknowledgments}

The authors would like to acknowledge Lorena LopezGonzalez and Isabelle Winer for analytic support. Editorial support was provided by The Lockwood Group, which was in accordance with Good Publication Practice (GPP3) and funded by AstraZeneca. 


\section{References}

1 Stockman LJ, Curns AT, Anderson LJ, Fischer-Langley G. Respiratory syncytial virus-associated hospitalizations among infants and young children in the United States, 1997-2006. Pediatr Infect Dis J 2012;31(01):5-9

2 Centers for Disease Control and Prevention. RSV in infants and young children. Available at: https://www.cdc.gov/rsv/high-risk/ infants-young-children.html. Accessed February 20, 2018

3 Boyce TG, Mellen BG, Mitchel EF Jr, Wright PF, Griffin MR. Rates of hospitalization for respiratory syncytial virus infection among children in Medicaid. J Pediatr 2000;137(06):865-870

4 Stevens TP, Sinkin RA, Hall CB, Maniscalco WM, McConnochie KM. Respiratory syncytial virus and premature infants born at 32 weeks' gestation or earlier: hospitalization and economic implications of prophylaxis. Arch Pediatr Adolesc Med 2000;154(01): 55-61

5 Ambrose CS, Anderson EJ, Simões EA, et al. Respiratory syncytial virus disease in preterm infants in the U.S. born at 32-35 weeks gestation not receiving immunoprophylaxis. Pediatr Infect Dis J 2014;33(06):576-582

6 Winterstein AG, Knox CA, Kubilis P, Hampp C. Appropriateness of age thresholds for respiratory syncytial virus immunoprophylaxis in moderate-preterm infants: a cohort study. JAMA Pediatr 2013; 167(12):1118-1124

7 Mauskopf J, Margulis AV, Samuel M, Lohr KN. Respiratory syncytial virus hospitalizations in healthy preterm infants: systemic review. Pediatr Infect Dis J 2016;35(07):e229-e238

8 Colin AA, McEvoy C, Castile RG. Respiratory morbidity and lung function in preterm infants of 32 to 36 weeks' gestational age. Pediatrics 2010;126(01):115-128

9 Langston C, Kida K, Reed M, Thurlbeck WM. Human lung growth in late gestation and in the neonate. Am Rev Respir Dis 1984;129 (04):607-613

10 Collins PL, Graham BS. Viral and host factors in human respiratory syncytial virus pathogenesis. J Virol 2008;82(05):2040-2055

11 Yeung CY, Hobbs JR. Serum-gamma-G-globulin levels in normal premature, post-mature, and "small-for-dates" newborn babies. Lancet 1968;1(7553):1167-1170

12 Synagis ${ }^{\circledR}$ (palivizumab) [prescribing information]. Gaithersburg, MD: AstraZeneca; 2014

13 American Academy of Pediatrics. Respiratory syncytial virus. In: Pickering LK, Baker CJ, Kimberlin DW, Long SS, eds. Red Book: 2012 Report of the Committee on Infectious Diseases, 29th ed. Elk Grove Village: American Academy of Pediatrics; 2012: 609-617

14 American Academy of Pediatrics Committee on Infectious Diseases; American Academy of Pediatrics Bronchiolitis Guidelines Committee. Updated guidance for palivizumab prophylaxis among infants and young children at increased risk of hospitalization for respiratory syncytial virus infection. Pediatrics 2014; 134(02):415-420

15 Anderson EJ, Krilov LR, DeVincenzo JP, et al. SENTINEL1: an observational study of respiratory syncytial virus hospitalizations among U.S. infants born at 29 to 35 weeks' gestational age not receiving immunoprophylaxis. Am J Perinatol 2017;34(01): 51-61

16 Rajah B, Sánchez PJ, Garcia-Maurino C, Leber A, Ramilo O, Mejias A. Impact of the updated guidance for palivizumab prophylaxis against respiratory syncytial virus infection: a single center experience. J Pediatr 2017;181:183-188

17 Blake SM, Tanaka D, Bendz LM, Staebler S, Brandon D. Evaluation of the financial and health burden of infants at risk for respiratory syncytial virus. Adv Neonatal Care 2017;17(04):292-298

18 Grindeland CJ, Mauriello CT, Leedahl DD, Richter LM, Meyer AC. Association between updated guideline-based palivizumab administration and hospitalizations for respiratory syncytial virus infections. Pediatr Infect Dis J 2016;35(07):728-732
19 Farber HJ. Impact of the 2014 American Academy of Pediatrics guidance on respiratory syncytial virus and bronchiolitis hospitalizations rates for infants born prematurely. J Pediatr 2017; $185: 250$

20 Kong AM, Krilov LR, Fergie J, et al. The 2014-2015 national impact of the 2014 American Academy of Pediatrics guidance for respiratory syncytial virus immunoprophylaxis on preterm infants born in the United States. Am J Perinatol 2018;35(02):192-200

21 Palmer L, Hall CB, Katkin JP, et al. Respiratory outcomes, utilization and costs 12 months following a respiratory syncytial virus diagnosis among commercially insured late-preterm infants. Curr Med Res Opin 2011;27(02):403-412

22 Palmer L, Hall CB, Katkin JP, et al. Healthcare costs within a year of respiratory syncytial virus among Medicaid infants. Pediatr Pulmonol 2010;45(08):772-781

23 Krilov LR, Masaquel AS, Weiner LB, Smith DM, Wade SW, Mahadevia PJ. Partial palivizumab prophylaxis and increased risk of hospitalization due to respiratory syncytial virus in a Medicaid population: a retrospective cohort analysis. BMC Pediatr 2014;14:261

24 McLaurin KK, Farr AM, Wade SW, Diakun DR, Stewart DL. Respiratory syncytial virus hospitalization outcomes and costs of full-term and preterm infants. J Perinatol 2016;36(11):990-996

25 Pavilack M, Clifford RA, Gonzales T, Kong AM, Wade S, McLaurin KK. Trends in utilization of outpatient respiratory syncytial virus prophylaxis with palivizumab among Medicaid- and commercially insured infants. Infect Dis Ther 2018;7 (01):121-134

26 Blanken MO, Rovers MM, Molenaar JM, et al; Dutch RSV Neonatal Network. Respiratory syncytial virus and recurrent wheeze in healthy preterm infants. N Engl J Med 2013;368(19): 1791-1799

27 Palivizumab, a humanized respiratory syncytial virus monoclonal antibody, reduces hospitalization from respiratory syncytial virus infection in high-risk infants. The IMpact-RSV Study Group. Pediatrics 1998;102(3 Pt 1):531-537

28 Feltes TF, Cabalka AK, Meissner HC, et al; Cardiac Synagis Study Group. Palivizumab prophylaxis reduces hospitalization due to respiratory syncytial virus in young children with hemodynamically significant congenital heart disease. J Pediatr 2003;143(04): 532-540

29 Anderson EJ, Carosone-Link P, Yogev R, Yi J, Simões EAF. Effectiveness of palivizumab in high-risk infants and children: a propensity score weighted regression analysis. Pediatr Infect Dis J 2017;36(08):699-704

30 Hall CB, Weinberg GA, Blumkin AK, et al. Respiratory syncytial virus-associated hospitalizations among children less than 24 months of age. Pediatrics 2013;132(02):e341-e348

31 Simões EA, Anderson EJ, Wu X, Ambrose CS. Effects of chronologic age and young child exposure on respiratory syncytial virus disease among US preterm infants born at 32 to 35 weeks gestation. PLoS One 2016;11(11):e0166226

32 Tregoning JS, Schwarze J. Respiratory viral infections in infants: causes, clinical symptoms, virology, and immunology. Clin Microbiol Rev 2010;23(01):74-98

33 AAP Updates Recommendations for Use of Palivizumab Against RSV [news release]. American Academy of Pediatrics; July 28, 2014. Available at: https://www.aap.org/en-us/about-the-aap/ aap-press-room/pages/AAP-Updates-Recommendations-for-Use -of-Palivizumab-Against-RSV.aspx. Accessed February 20, 2018

34 American Academy of Pediatrics Committee on Infectious Diseases; American Academy of Pediatrics Bronchiolitis Guidelines Committee. Updated guidance for palivizumab prophylaxis among infants and young children at increased risk of hospitalization for respiratory syncytial virus infection. Pediatrics 2014; 134(02):e620-e638

35 Ambrose CS. Statistical power to detect an association between guideline-based palivizumab administration and hospitalizations 
1442 RSV Hospitalizations among U.S. Preterm Infants Compared with Term Infants Goldstein et al.

for respiratory syncytial virus infections. Pediatr Infect Dis J 2017; 36(03):348

36 Texas Medicaid/CHIP Vendor Drug Program Fee-For-Service Medicaid Synagis ${ }^{\circledR}$ Request Form, 2014-15 Season. Available at: http://www.maxor.com/forms/IVSolutions/pdfs/lubbock/synagis/SMN-Tx\%20Medicaid\%20Vendor\%20Drug\%20091614-IVSL. pdf. Accessed February 20, 2018
37 Makari D, Staat MA, Henrickson KJ, Wu X, Ambrose CS. The underrecognized burden of respiratory syncytial virus among infants presenting to US emergency departments. Clin Pediatr (Phila) 2015;54(06):594-597

38 Ralston SL, Lieberthal AS, Meissner HC, et al; American Academy of Pediatrics. Clinical practice guideline: the diagnosis, management, and prevention of bronchiolitis. Pediatrics 2014;134(05):e1474-e1502 